\title{
Preparation of Polystyrene/Polydopamine/Ag/ Polydopamine Microspheres with Multilayer Core-Shell Structure and Its Alignment in Dimethyl Silicone Oil Induced by Electric Field
}

\author{
Zhifeng Wang ${ }^{* a, b}$, Zhikun Chen ${ }^{\mathrm{a}}$, Ming Zhang ${ }^{* a, b}$ \\ a School of Chemistry and Chemical Engineering, Yangzhou University, Yangzhou 225002, China \\ ${ }^{\mathrm{b}}$ Testing Center, Yangzhou University, Yangzhou 225009, China \\ *Corresponding Author: zfwang@yzu.edu.cn, lxyzhangm@yzu.edu.cn
}

\begin{abstract}
PSt/PDA (Polystyrene/Polydopamine) microspheres were synthesized by polydopamine coating on PSt microspheres. Ag nanoparticles were then deposited on these microspheres surface. A layer of PDA was coated on the surface of PSt/PDA/Ag to weaken the conductivity of composites microspheres. PSt/PDA/Ag/PDA multilayer microspheres were confirmed by transmission electron microscopy, X-ray photoelectron spectroscopy, and high-resolution transmission electron microscope. These microspheres were dispersed into dimethyl silicone oil under an electric field and the alignment behavior of multilayer microspheres were observed. The results showed that the alignment regularity of microspheres increased with the concentration of microspheres and the strength of the electric field.
\end{abstract}

Keywords: PSt/Ag composite microspheres; Core-shell structure; Field polarization

\section{Introduction}

Composite conductive polymer microspheres has been widely investigated owing to their good performances of both polymers and metals ${ }^{[1-4]}$. Dielectric elastomer possesses some characteristic advantages, including the high elasticity, high dielectric constant and lower modulus. Dielectric elastomer could produce a large deformation under electric field which could achieve the conversion between electrical energy and mechanical energy. Dielectric elastomer filled with conductive polymeric microspheres have attracted a lot of attention because of the remarkable enhancement of dielectric constant with lower modulus. ${ }^{[5-8]}$ The dielectric particles which were prepared from conductive composite microspheres can be aligned to form ordered structure by the electric field polarization. ${ }^{[9-10]}$ Herein, the preparation of PSt/PDA/Ag/PDA composite dielectric microspheres and the alignment behavior of multilayer microspheres under electric field were investigated.

\section{Experimental}

\subsection{Experimental materials}

Polystyrene microspheres with a diameter of $753 \mathrm{~nm}$ were synthesized in our lab. Dopamine hydrochloride(DA) was purchased from Alfa Aesar company, USA. Tris(hydroxymethyl)-amino-methane (Tris), silver nitrate(AgNO3) and polyvinylpyrrolidone (PVP) were bought from Sinopharm Chemical Reagent Co., Ltd (Shanghai, China). Dimethyl silicone oil was obtained from Jiang Yang West Silicone Co., Ltd.

\subsection{Preparation of PSt/PDA microspheres}

DA solution: Dopamine hydrochloride $(0.04 \mathrm{~g})$ was dissolved into distilled water $(20 \mathrm{~mL})$ to obtain DA solution(2 $\mathrm{g} / \mathrm{L})$, then dropping Tris solution(2 g/L) into DA solution until the $\mathrm{pH}$ of solution was 8.5.

Preparation of PSt/PDA microspheres: Dried PSt microspheres $(0.1 \mathrm{~g})$ was dispersed in DA solution $(50 \mathrm{~mL})$ under ultrasonic, then reacted for 24 hours under magnetic stirring at room temperature.

\subsection{Preparation of PSt/PDA/Ag composite conductive microspheres}

PSt/PDA microspheres were dispersed in the silver 


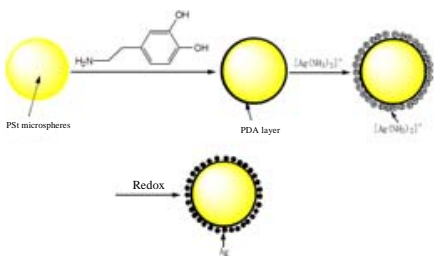

Fig. 1 Schematic illustration of procedure for preparation of PSt/PDA/Ag composite microspheres

ammonia solution, then reacted for about $1 \mathrm{~h}$ under stirring(300 rpm) at room temperature. It was continued stirring $1 \mathrm{~h}$ by placing in an oil bath at $70{ }^{\circ} \mathrm{C}$. The $\mathrm{PSt} / \mathrm{PDA} / \mathrm{Ag}$ composite microspheres were obtained.

\subsection{Preparation of PSt/PDA/Ag/PDA dielectric particles}

PSt/PDA/Ag/PDA composite dielectric particles were obtained by coating PSt/PDA/Ag composite microspheres with a layer of PDA.

\subsection{Alignment of dielectric particles under electric field}

PSt/PDA/Ag/PDA composite dielectric particles were used as filler, and dimethyl silicone oil was used as dispersed phase. Then they were mixed and placed under electric field.

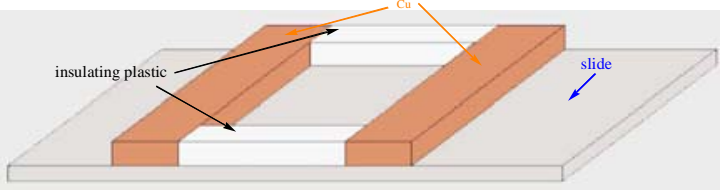

Fig. 2 Sketch of experiments mold for alignment of dielectric particles under electric field

\section{Characterization}

Transmission electron microscopy (TEM) observation was performed on a Tecnai 12 electron microscope with an operating voltage of $120 \mathrm{kV}$. Elemental mapping images were acquired by energy-dispersive X-ray spectroscopy (EDS) using a Tecnai G2 F30 S-TWIN electron microscope equipped with a scanning transmission electron microscopy (STEM) unit and an Inca Energy 250 detector. X-ray photoelectron spectroscopy (XPS) was applied to detect elements and valence distribution. The alignment of dielectric particles were observed by optical microscope.

\section{Results and Discussion}

\subsection{Characterization of Morphology and Structure of PSt/PDA/Ag/PDA Composite Microsphere}

TEM images of PSt/PDA/Ag/PDA composite microspheres are shown in Fig.3. Ag nanoparticles can be observed on the surface of microspheres, as shown in Fig.3(a). The high magnification image in Fig.3 (b) shows that there is a layer of material outside Ag particles, which were inferred as PDA.

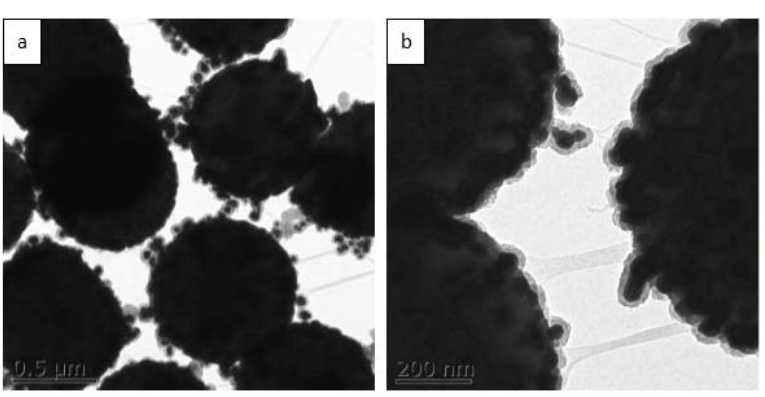

Fig. 3 TEM images of PSt/PDA/Ag/PDA Composite Microspheres a)13500X, b)59000X
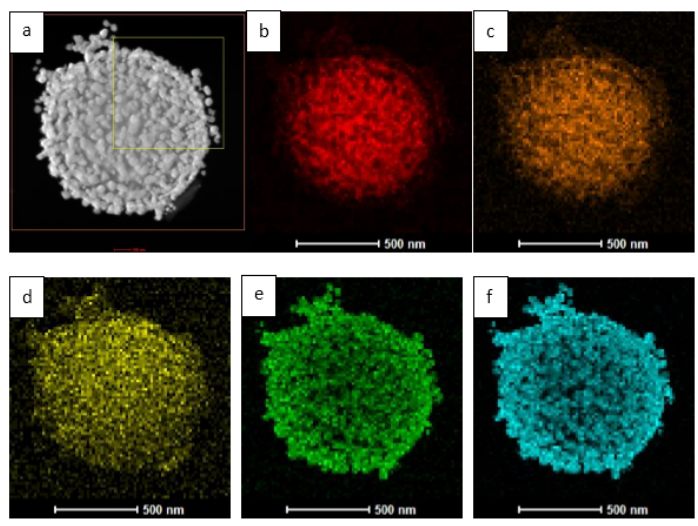

Fig. 4 a) HAADF-STEM and b-f) EDS of PSt/PDA/Ag/PDA composite microsphere

a) HAADF-STEM, b) C-K, c) N-K, d) O-K, e) Ag$\mathrm{K}$, f) Ag-L

To confirm the Ag nanoparticles and PDA layer, HRTEM was employed. As shown in Fig. 4, the element component of microsphere is $\mathrm{C}, \mathrm{N}, \mathrm{O}$ and $\mathrm{Ag}$. Combining Fig. 4 and Table 1, it can be found that $\mathrm{C}$ and $\mathrm{N}$ mainly distributes in the inner layer, while Ag exists in the middle layer of composite microsphere. Furthermore, $\mathrm{O}$ distributes in the outermost layer which comes from dopamine. It indicates that the outermost layer of composite microsphere is PDA layer.

Table 1 The average diameters calculated from the profiles of elements distribution in HRTEM

\begin{tabular}{cccccc}
\hline Element & C-K & N-K & O-K & Ag-K & Ag-L \\
\hline $\begin{array}{c}\text { Diameter } \\
\text { /nm }\end{array}$ & 846.64 & 873.18 & 1004.1 & 989.71 & 985.69 \\
\hline
\end{tabular}

X-ray photoelectron spectroscopy was also applied to determine the core-shell structure of PSt/PDA/Ag/PDA composite microsphere. Fig.5 a) shows that PSt/PDA/Ag/ 
PDA composite microsphere consists of the elements of $\mathrm{C}$, $\mathrm{N}, \mathrm{O}$ and $\mathrm{Ag}$. The theoretical ratios of atom number of $\mathrm{C} / \mathrm{N}$, $\mathrm{C} / \mathrm{O}$ and $\mathrm{O} / \mathrm{N}$ in dopamine are 8, 4 and 2, respectively. In this case, the ratios of atom number of $\mathrm{C} / \mathrm{N}, \mathrm{C} / \mathrm{O}$ and $\mathrm{O} / \mathrm{N}$ are more higher than the theoretical ratios of DA, equaling to 14.2, 6.1 and 2.3, respectively. The extra atom number is considered as the contribution of polystyrene. It implies that the thickness of PDA layer is less than $10 \mathrm{~nm}$, and the $\mathrm{Ag}$ layer is not compact, which agrees with TEM images in Fig.3. As seen from Fig. 5 b), there are four peaks in the $C$ 1s spectrum, C-C, C-N, C-O and C=O, respectively. It indicates that there is a PDA layer. As shown in Fig.5 c), Ag 3d spectrum consists of two peaks, Ag 3d3/2 and Ag 3d5/2. The difference of the binding evergies is $6 \mathrm{eV}$, which corresponds to the zero-valent silver. It proves that the $\mathrm{Ag}$ on the surface of $\mathrm{PSt} / \mathrm{PDA} / \mathrm{Ag}$ composite microspheres is elementary substance.

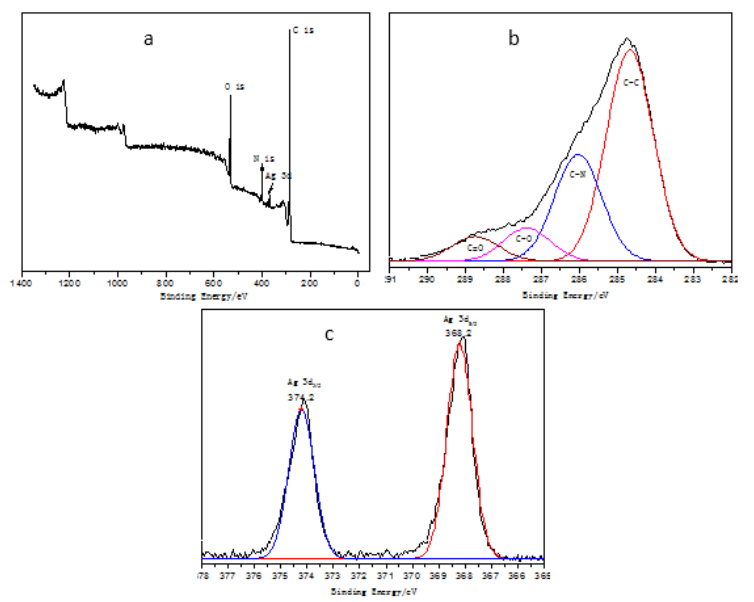

Fig. 5 XPS spectras of PSt/PDA/Ag/PDA composite microspheres

a) XPS full-spectrum, b) C 1s spectrum, c) Ag 3d spectrum

\subsection{Alignment of PSt/PDA/Ag/PDA dielectric particles under electric field}

The behavior of alignment of composite dielectric particles in dimethyl silicone oil under electric field was observed via optical microscope. Fig. 6 shows the images of aligned dielectric particles at different concentrations under electric field. As seen from Fig. 6(a1)-(a6), the dielectric particles randomly distribute in the dimethyl silicone oil without electric field inducement. However, the alignment of dielectric particles appears under electric field, as shown in Fig. 6(b1)-(b6). Compared with Fig. 6(b1)-(b3), the dielectric particles were aligned and form a continuous ordered structure in Fig. 6(b4)-(b6).
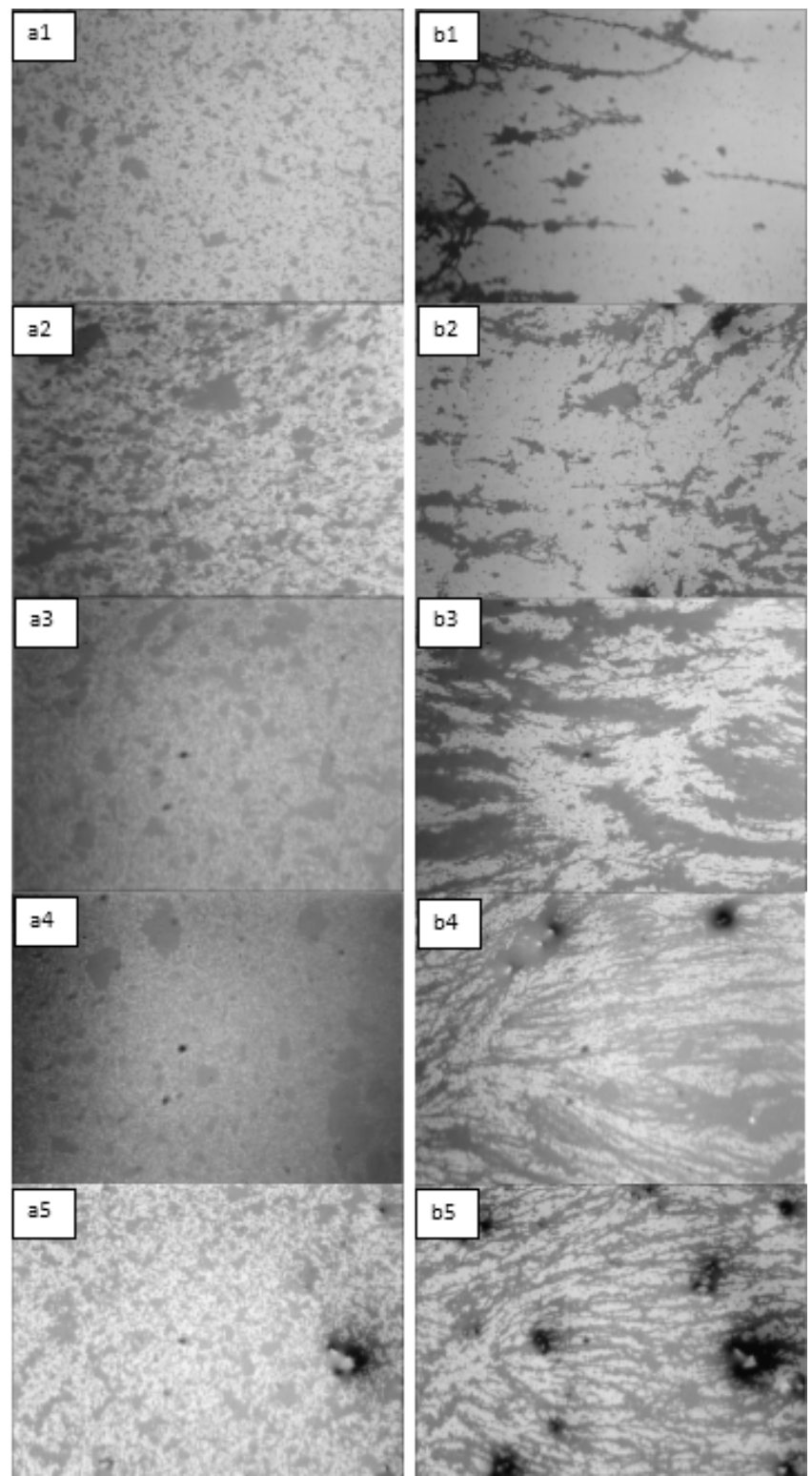

b2
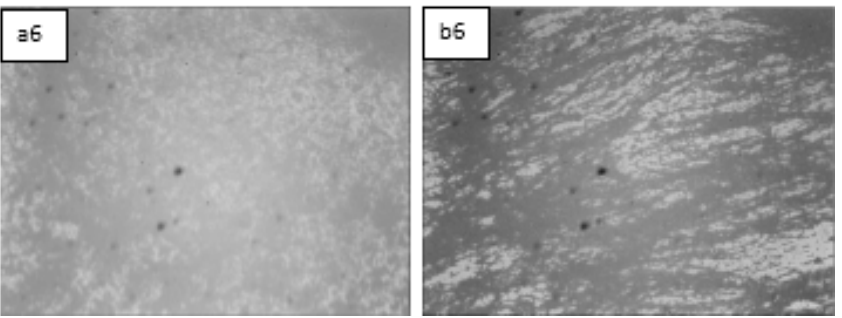

Fig. 6 Optical microscope images of dielectric particles with different concentrations in dimethyl silicone oil under electric field $(E=150 \mathrm{~V} / \mathrm{mm})$

a) $0 \mathrm{~min}$, b) $30 \mathrm{~min}$

1) $1 \%$, 2) $3 \%$, 3) $5 \%$, 4) $7 \%$, 5) $10 \%$, 6) $20 \%$

Dielectric particles can be arrayed due to the polarization effect of electric field. When the amount of particles is less, there are some blank areas after alignment of particles. These blank areas can't be filled without more dielectric particles. So with increasing the particle 
concentration, the alignment regularity of particles gets better.

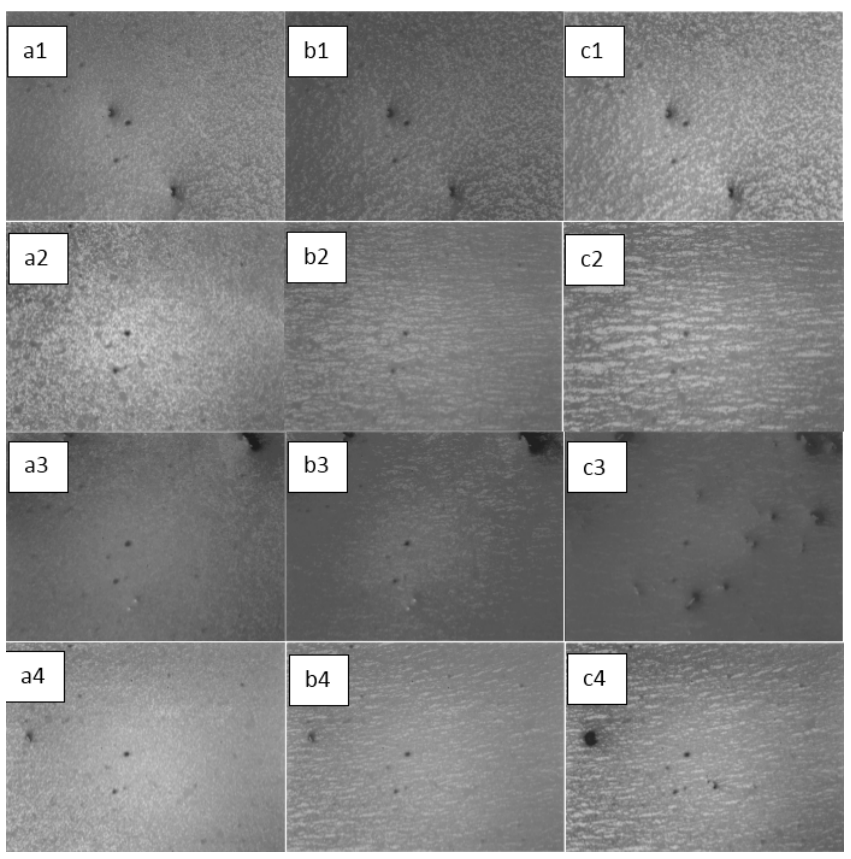

Fig 7 Optical microscope images of arrangement of particles under different electric field strength

a) $0 \mathrm{~min}$, b) $10 \mathrm{~min}$, c) $30 \mathrm{~min}$

1) $50 \mathrm{~V} / \mathrm{mm}$, 2) $100 \mathrm{~V} / \mathrm{mm}$, 3) $150 \mathrm{~V} / \mathrm{mm}$, 4) $200 \mathrm{~V} / \mathrm{mm}$

The formation of ordered structure under electric field was also investigated. Under the lower electric field $(\mathrm{E}=50 \mathrm{~V} / \mathrm{mm})$, the dielectric particles distribute disorderly after 30min in Fig.7(c1). As seen from Fig.7(c2)-(c4), the chain-like distribution of dielectric particles is found after 30min with the inducement of electric field. Furthermore, with the increasing of the electric field strength, the time needed to form chain becomes shorter, as shown in Fig.7(b2)-(b4). These results could be attributed to the increase of polarization force with increasing the electric field strength. The stronger polarization force between particles is, the easier chain-like distribution forms.

\section{Conclusions}

PSt/PDAAg/PDA core/shell dielectric particles were synthesized by dopamine surface coating. These dielectric particles were dispersed in dimethyl silicone oil and their alignment behavior under electric field were investigated. The results show that the dielectric particles can form the chain-like distribution under electric field. The alignment regularity of dielectric particles increases with increasing the filling amount and the electric field strength.

\section{Acknowledgment}

Authors acknowledge the support by National Science Foundation of China (No. 51403181).

\section{References}

(1) Yuan L., et al., Mussel-inspired biomimetic surface functionalization and metallization[D]. Beijing University of Chemical Technology, 2010.

(2) Cong, Y., et al., Mussel-inspired polydopamine coating as a versatile platform for synthesizing polystyrene/Ag nanocomposite particles with enhanced antibacterial activities. Journal Of Materials Chemistry B, 2014. 2(22): p. 3450-3461.

(3) Xi Z Y, Xu Y Y, Zhu L P. A facile method of surface modification for hydrophobic polymer membranes based on the adhesive behavior of poly(DOPA) and poly(dopamine)[J]. Membrane Sci., 2009, 327: 244-253.

(4) Li L H., et al., Preparation of metal-coated composite particles of conductive polymer[D]. Nanjing University of Science and Technology, 2007.

(5) Yu J X., et al., Study on preparation and properties in silicone matrix electroactive dielectric elastomer composites[D]. Beijing University of Chemical Technology, 2011.

(6) Hang Z., et al., Performance characterization and actuation behavior simulation of flexible silicone based dielectric elastomer composites[D]. Beijing University of Chemical Technology, 2012.

(7) Hao L L et al., The preparation and study of silicone dielectric elastomer composites[D]. Beijing University of Chemical Technology, 2012.

(8) Yang D, Tian M, Wang W, et al. Controllable dielectric and electrical performance of polymer composites with novel core/shell-structured conductive particles through biomimetic method[J]. Electrochimica Acta, 2013, 87(1):9-17.

(9) Park C, Robertson R E. Mechanical properties of resin composites with filler particles aligned by an electric field.[J]. Dental Materials Official Publication of the Academy of Dental Materials, 1998, 14(6):385-93.

(10) William M. Chirdon, William J. O'Brien, Richard E. Robertson. Fraunhofer diffraction of short-fiberreinforced composites aligned by an electric field[J]. Dental Materials, 2006, 22(2):107-111. 